\title{
Comparative Study of Biopolymer Flooding: A Core Flooding and Numerical Reservoir Simulator Validation Analysis
}

\author{
Akinleye Sowunmi, ${ }^{1}$ Vincent E. Efeovbokhan ${ }^{(D)},{ }^{1}$ Oyinkepreye D. Orodu $\mathbb{D}^{2}{ }^{2}$ \\ Oluwasanmi Olabode $\left(\mathbb{D},{ }^{2}\right.$ and Alma Oputa ${ }^{3}$ \\ ${ }^{1}$ Department of Chemical Engineering, Covenant University, P.M.B 1023, Ota, Ogun State, Nigeria \\ ${ }^{2}$ Department of Petroleum Engineering, Covenant University, P.M.B 1023, Ota, Ogun State, Nigeria \\ ${ }^{3}$ Department of Business Management, Covenant University, P.M.B 1023, Ota, Ogun State, Nigeria
}

Correspondence should be addressed to Oluwasanmi Olabode; oluwasanmi.olabode@covenantuniversity.edu.ng Received 13 November 2021; Accepted 31 January 2022; Published 24 February 2022

Academic Editor: Houari Ameur

Copyright (C) 2022 Akinleye Sowunmi et al. This is an open access article distributed under the Creative Commons Attribution License, which permits unrestricted use, distribution, and reproduction in any medium, provided the original work is properly cited.

\begin{abstract}
Polymers increase the macroscopic efficiency of the flooding process and increase crude oil recovery. The viscosity of 3 polymers xanthan, guar, and Arabic gums is measured in the lab and experimented with as EOR options. Xanthan and guar gum polymers are measured in weight percentages of $0.1,0.2,0.2,0.4,0.5$, and 1 , while gum Arabic is measured in $0.4,0.5,1,5,10$, and 15 weight percentages. The viscosity experiments showed that gum Arabic had the lowest viscosity at 15\% wt. Xanthan gum and guar gum had significantly higher viscosities than gum Arabic at corresponding weight percentages. At the same weight of $0.5 \%$, xanthan, guar, and Arabic gums recorded a 63\%,53\%, and 46\% oil recovery, respectively. Due to the limitations surrounding core flooding experiments such as human error, equipment failure, and measurement of oil recoveries, it is necessary to validate the results obtained with other methods such as reservoir simulation. A reservoir model is built (using Eclipse) and incorporated with polymer and viscosity functions measured in the lab to validate results from the core flooding experiments. Peak oil recovery of $9.96 \%, 9.95 \%$, and $9.90 \%$ was recorded for xanthan, guar, and Arabic gum, respectively, at a weight percentage of $0.5 \%$ weight. Also, increasing the wt\% of injected polymers increases oil recovery. Results also indicate that the trend of oil recoveries during core flooding follows that observed during reservoir simulation and oil production increased as percentage weight increased for all the polymer cases considered.
\end{abstract}

\section{Introduction}

The demand of energy to sustain an increase in world population and industrial expansion has been on the increase. Unfortunately, the capital costs and risks involved in venturing into exploitation of newer reserves are high and existing reserves at plateau production. For reservoirs with medium to heavy crude oils, these natural production conditions are severe due to the high viscous nature of the crude oil.

Thermal options like in situ combustion, hot water flooding, and steam flooding described by (Cheraghian, [1]) are usually expensive (especially for facilities), which results in scale formation in wellbore tubing and creates a possible adverse effect on the reservoir and environment. Thermal options when implemented in medium-heavy crude reservoir reduce the oil viscosity enabling easier flow but with loss in the lighter component of the hydrocarbon, loss of heat in highly fractured reservoirs, and possible reduction in reservoir permeability due to coke formation during in situ combustion [2]. These issues coupled with the costs and availability of gas and steam injection have led to other options for enhanced oil recovery.

Chemical options for enhanced oil recovery are preferred but must be screened and ranked based on reservoir rock and fluid properties, costs of implementation, environmental impact, and expected recovery factors [3, 4]. Applications of chemical EOR options vary with respect to the prevailing reservoir fluids and rock properties and involve various mechanisms such as reducing interfacial tension [5], altering surface wettability [6], mobility control by using high viscosity agents such as polymers [7], and application of microbes for oil recovery of depleted reservoirs [8]. EOR 

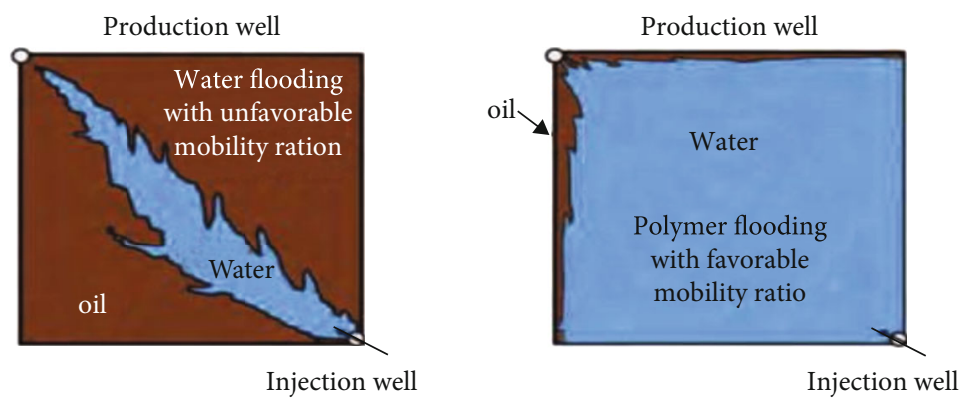

FIGURE 1: Schematic of water and polymer flood [11].

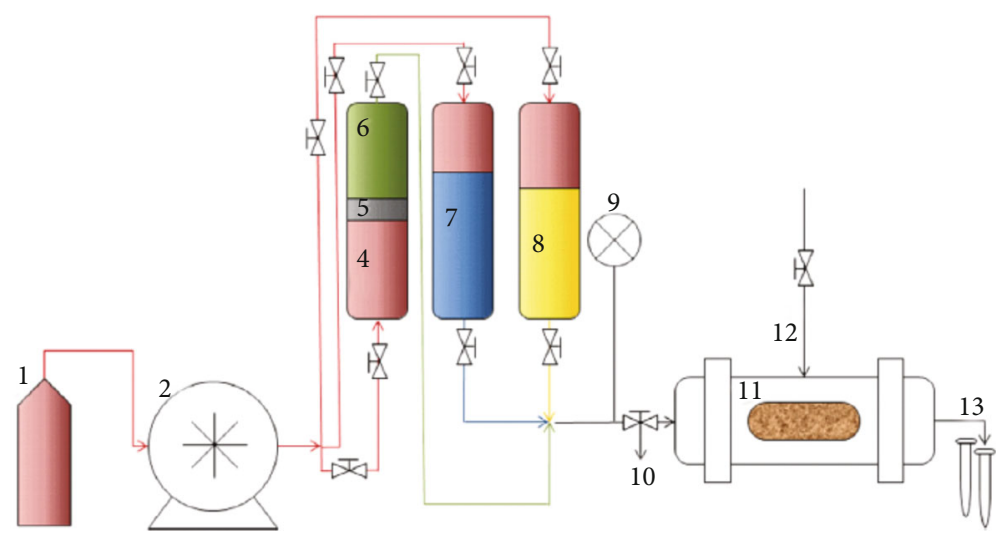

FIGURE 2: RPT schematic setup.

processes can include one or more of these mechanisms, and to be successful, the methods must be profitable, practical, and reliable [9].

The natural option to first consider is water flooding for reservoirs with medium-heavy crude. The challenge is that the injected water moves faster than the crude oil due to its lower viscosity. This leads to an irregular flood front (Figure 1) that leaves behind unswept zones [10]. Adding polymers to injected water increases its viscosity and reduces the permeability to water due to entrapment of the polymer, thus reducing its mobility and creating a uniform sweep.

Chemical enhanced oil recovery (EOR) is one of the most common EOR methods. It involves the injection of chemicals such as such as surfactant, alkaline, and polymer into a reservoir to increase crude oil recovery from the reservoir either by microscopic or macroscopic interactions. Surfactants are surface-active agents that improve oil recovery by reducing the interfacial tension (IFT) between crude oil and connate water. Alkaline is normally used in combination with surfactant and polymer in flooding experiments to reduce the adsorption of the surfactant to the rock matrix $[11,12]$.

Polymers are used to reduce the mobility ratio of the flooding process to achieve greater macroscopic efficiency. Polymers may be used as sole injection fluid in chemical EOR or in combination with surfactants and alkaline. The most used natural and synthetic polymers used in EOR are xanthan gum and hydrolyzed polyacrylamide, respectively.
Factors such as reservoir permeability, oil viscosity, polymer retention, and adsorption on rock surfaces are essential factors to consider for a successful polymer flooding type and option (Olajire, [13] \& [14]).

Costs incurred during large field scale deployment of polymers, high adsorption rates, and adverse environmental impacts have led to the advent of biopolymers (BP) for enhanced oil recovery processes. Nowadays, biopolymers are extracted from waste food materials such as potatoes peels [15] and banana peels and mango kernel [16] through an esterification and carboxymethylation [17].

In this work, xanthan gum was used alongside two other natural polymers, guar gum and gum Arabic, in core flooding experiments and in a numerical reservoir simulator. Xanthan gum contains D-glucuronic acid, D-mannose, and D-glucose and is used in different industries including textile, food, and pharmaceuticals [18]. Xanthan gum polymer was tested in EOR experiment for heavy oil recovery at $90^{\circ} \mathrm{C}$ and high brine concentration of $244.121 \mathrm{mg} / \mathrm{L}$. The results showed that xanthan gum achieved oil recovery of $9.5 \%$ of original oil in place (OOIP) [19]. Other studies have shown xanthan gum to have oil recovery potential up to $18 \%$ of incremental recovery [20]. Guar gum is used as a thickener and stabilizer, and it therefore has good potential as a viscosity enhancer in EOR. Guar gum was used in EOR for flooding of a sand pack sample, and it achieved a total recovery of $55.1 \%$ and incremental recovery of $15.76 \%$ [21]. Gum Arabic is a low viscosity 
TABLE 1: PVT properties of water.

\begin{tabular}{lc}
\hline Property & Quantity \\
\hline Reference pressure $(\mathrm{atm})$ & 0 \\
Water FVF* at $\mathrm{P}_{\text {ref }}(\mathrm{rcc} / \mathrm{scc})$ & 1 \\
Water compressibility $(1 / \mathrm{atm})$ & $3.03 \times 10^{-6}$ \\
Water viscosity at Pref $(\mathrm{cP})$ & 0.5 \\
Water viscosibility $(1 / \mathrm{atm})$ & 0
\end{tabular}

${ }^{*}$ Formation volume factor.

TABLE 2: PVT properties of dead oil (no dissolved gas).

\begin{tabular}{lcc}
\hline Pressure $(\mathrm{atm})$ & FVF $(\mathrm{rcc} / \mathrm{scc})$ & Viscosity $(\mathrm{cP})$ \\
\hline 0 & 1 & 2 \\
8000 & 0.92 & 2 \\
\hline
\end{tabular}

TABle 3: Saturation functions of water and oil.

(a)

\begin{tabular}{lcc}
\hline $\begin{array}{l}\text { Water saturation } \\
\left(\mathrm{S}_{\mathrm{w}}\right)\end{array}$ & $\begin{array}{c}\text { Relative permeability to } \\
\text { water }\left(\mathrm{K}_{\mathrm{rw}}\right)\end{array}$ & $\begin{array}{c}\text { Capillary } \\
\text { pressure }\left(\mathrm{P}_{\mathrm{c}}\right)\end{array}$ \\
\hline 0.25 & 0 & 4 \\
0.7 & 1 & 0 \\
\hline
\end{tabular}

(b)

\begin{tabular}{lc}
\hline Oil saturation $\left(\mathrm{S}_{\mathrm{o}}\right)$ & Relative permeability to oil $\left(\mathrm{K}_{\mathrm{ro}}\right)$ \\
\hline 0.3 & 0 \\
0.75 & 1 \\
\hline
\end{tabular}

polymer obtained from Acacia tree from the leguminosae specie. Although gum Arabic has limited application in EOR, it is found in abundant quantities in Sudan, Chad, Senegal, Ethiopia, and Nigeria, and it has great potential because it is cheap and possesses both polymer and surfactant properties [22]. These polymers were tested to determine their comparative recoveries both at laboratory and field scale to provide insight into the possibility of upscaling them to field scale applications.

Important considerations in the application chemical EOR are cost and the duration of conducting experiments which are susceptible to human error. For this reason, results obtained usually need a second-hand evaluation and validation before field applications. A reservoir simulation tool such as Eclipse has various functions including modelling reservoirs for polymer flooding and optimizing production parameters such as well lengths, production, and injection rates as discussed by Olabode et al., (2018) [23]. Also, it allows for sensitivity analysis and optimization of parameters that affects polymer injections such as viscosity, temperature, injection rates, concentrations, and incorporation of a 3 fluid phase model (oil, water, and gas) which ulti-
TABLE 4: Polymer rock properties.

\begin{tabular}{lc}
\hline Property & Quantity \\
\hline Dead pore space & 0.16 \\
Residual resistance factor & 1.5 \\
Rock mass density $\left(\mathrm{g} / \mathrm{cm}^{3}\right)$ & 1000 \\
Adsorption index & 1 \\
Max polymer adsorption value & 0.001 \\
\hline
\end{tabular}

mately helps to predict water and gas productions (Olabode, et al., 2019) [24]. In his study, outlined factors affect oil recovery during polymer injection. The author discussed that a higher polymer injection would increase oil recovery and lower polymer production due to adsorption. But studies from $[25,26]$ do not suggest an ever-increasing oil recovery as polymer concentration increase. The author described a situation of maximum oil recovery at a threshold polymer concentration.

Before the field implementation of enhanced oil recovery options, it is essential that experimental analysis be considered. But due to human and equipment error coupled with integrity loss in material and data during the experiment, other tools such as reservoir simulation can be used to augment and support the investigated results during the experiments. Simulation studies on polymer flooding using a software tool such as Eclipse and Travigator have been studied to quantify the effect of polymer concentration, injection rates, and slug sizes on oil recovery. These tools can be used to perform other enhanced oil recovery functions such as low salinity flooding [27] and surfactant injection [28] for medium to heavy crude reservoirs. This research scope is validatory; thus, the chemistry entailing oil recovery during polymer flooding such polymer adsorption and retention is not covered. The aim of this research is to estimate oil recovery factor from experimental core flooding procedures using 3 different biopolymers and compare these results with those obtained from performing the same procedure using a reservoir model. This is essential to determine if similar trends and validations are observed before proper field implementation of polymer flooding.

\section{Materials and Methods}

2.1. Materials and Equipment. Three polymers were used in the experiments: gum Arabic, xanthan gum, and guar gum. Guar gum and xanthan gum were sourced from Ojota Chemical Market in Lagos, Nigeria, while the gum Arabic polymer was sourced from Panteka Market in Kaduna, Nigeria. Medium crude oil with viscosity of $14.5 \mathrm{cP}$ and API of $24.8^{\circ}$ was sourced from the Niger-Delta. Sandstone core plugs were also purchased from the Niger-Delta. Toluene (HPLC grade) manufactured by Fisher Chemical was used for cleaning the core plugs. The following equipment were used during the experiments: a Model 800 OFITE viscometer, a Soxhlet extractor, a manual saturator, and an OFITE Reservoir Permeability Tester (RPT). 

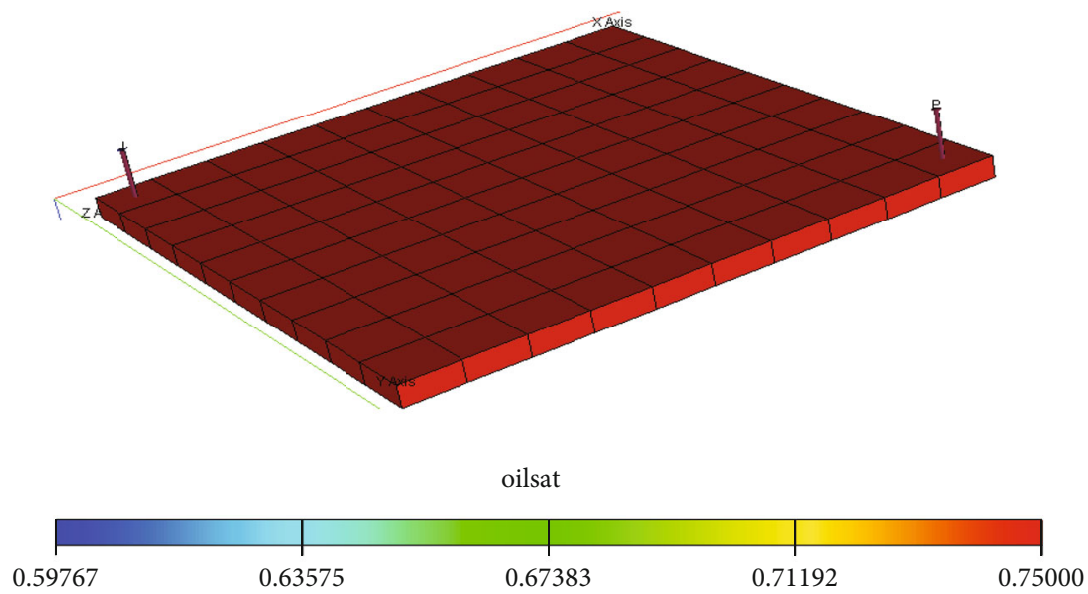

FIgURE 3: Start-up model with initial PVT and solution properties.

2.2. Experimental Procedures. Rheology experiments were carried out for xanthan gum, guar gum, and gum Arabic to determine viscosities at different polymer concentrations. The viscometer used a bob and rotor mechanism for viscosity measurement and was fitted with a dial screen that allowed for easy recording of readings that were converted to viscosity values. Polymers were mixed with deionized water to form polymer fluids. The viscosities of 6 different weight percentages were determined for each polymer. For xanthan gum and guar gum, the following weight percentages were used: $0.1,0.2,0.3,0.4,0.5$, and $1.0 \% \mathrm{w} / \mathrm{w}$. Higher weight percentages could not be used because of the high viscosities of both polymers. The highest weight percentage used for both polymers in the flooding experiments was 0.5 to avoid blocking the flow lines of the RPT. The viscosity of gum Arabic was done at 0.4, $0.5,1.0,5.0,10.0$, and $15 \% \mathrm{w} / \mathrm{w}$. Gum Arabic is a low viscosity polymer, and therefore, lower weight percentages were not used due to practical limitations. Its highest weight percentage was chosen based on literature values and laboratory try-outs. Viscosity was measured using the OFITE 800 Model Viscometer. The viscometer calibration was done according to the recommended API practice 13B-1 and 13B-2, respectively. The fluid to be measured was prepared and loaded into the sample cup up to the fill line. The viscometer sleeve was screwed unto the rotor, and the viscometer was operated in the air for about three minutes to loosen up the gears and bearings; this was only done on the first use for the day. The sample cup was placed on the platform and raised to the fill line of the viscometer sleeve. The viscometer was turned on; the knob was turned "STIR" setting for about 10 seconds to stir the fluid. The knob was then turned to the required shear rates, and readings were taken. The sample cup was lowered and emptied, and the sleeve and bob were cleaned before another measurement was taken. All viscosity measurements were done at room temperature.

A Soxhlet extractor was used for cleaning the core plugs to remove oil and other impurities from the core plugs. This was done using toluene as solvent. After cleaning the core plugs, the manual saturator was used to saturate the core
TABLE 5: Viscosity of polymers at different weight percentages.

\begin{tabular}{lccccc}
\hline \multicolumn{2}{c}{ Xanthan gum } & \multicolumn{2}{c}{ Guar gum } & \multicolumn{2}{c}{ Gum Arabic } \\
wt\% & Viscosity $(\mathrm{cP})$ & wt\% & Viscosity $(\mathrm{cP})$ & wt\% & Viscosity $(\mathrm{cP})$ \\
\hline 0.1 & 165 & 0.1 & 155 & 0.4 & 150 \\
0.2 & 245 & 0.2 & 205 & 0.5 & 170 \\
0.3 & 440 & 0.3 & 270 & 1 & 180 \\
0.4 & 450 & 0.4 & 390 & 5 & 200 \\
0.5 & 540 & 0.5 & 410 & 10 & 220 \\
1 & 1645 & 1 & 1510 & 15 & 245 \\
\hline
\end{tabular}

TABle 6: Properties of core plugs used for polymer flooding.

\begin{tabular}{lccc}
\hline Property & \multicolumn{3}{c}{ Value/label } \\
Core sample & XG & GG & GA \\
\hline Permeability $(\mathrm{md})$ & 298.15 & 248.15 & 145.8 \\
Porosity & 0.29 & 0.29 & 0.29 \\
Length $(\mathrm{cm})$ & 4.4 & 4.4 & 4.4 \\
Diameter $(\mathrm{cm})$ & 2.2 & 2.2 & 2.2 \\
\hline
\end{tabular}

plugs to determine the porosity and the permeability of the core plugs. The RPT was used to determine the permeability of the core plugs and to carry out the flooding experiments. The RPT (Figure 1) is made up of three chambers (points 6, 7 , and 8) that hold crude oil, brine, and the displacing fluid (polymer in this case); these chambers have inlet and outlet valves (connected to the core holder). It also has a compartment that holds the core plug (point 11) using a rubber sleeve and stainless-steel spacers for the flooding process. The valve and pumps (points 10 and 9) are used to control the flow of fluids from the 3 chambers to the core plug compartment. The core plug was first flooded with brine to create initial reservoir condition. This injection was done at $3 \mathrm{~cm}^{3} / \mathrm{min}$. The core was then injected with oil (which was earlier pumped into the accumulator) at $3 \mathrm{~cm}^{3} / \mathrm{min}$ until 


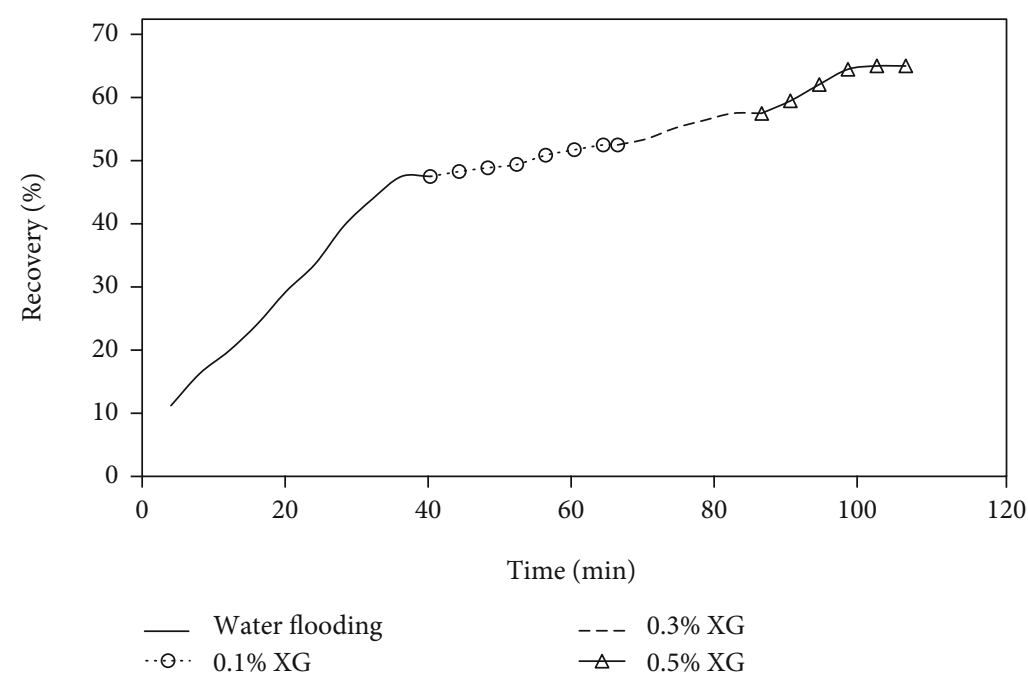

FIGURE 4: Total oil recovery for xanthan gum polymer.

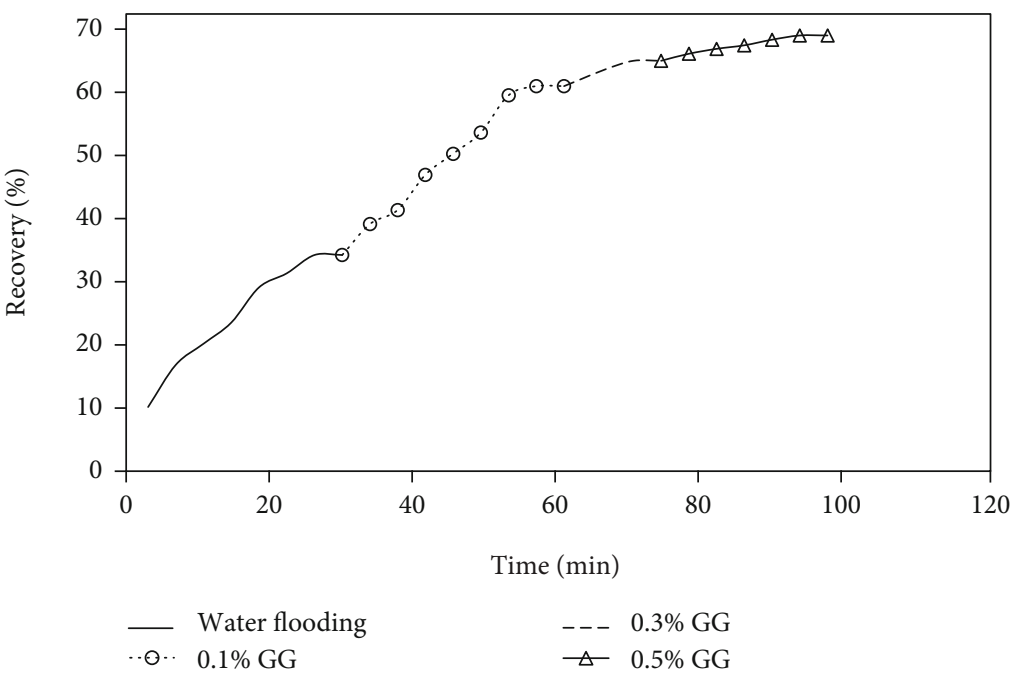

Figure 5: Total oil recovery for guar gum polymer.

the water saturation dropped from its maximum to connate water content to establish the irreducible water saturation. The core flooding process began with water flooding (base case) and then different polymers and their concentrations. During the flooding process, the amount of effluent collected (oil and water) at point 13 in Figure 2 is measured and recorded in a calibrated test tube at every 4-minute interval.

2.3. Reservoir Simulation. The EOR potential of six weight percentages of the three polymers was investigated using Schlumberger Eclipse ${ }^{\circledR}$ software. For guar gum and xanthan gum, weight percentages of $0.1,0.2,0.3,0.4,0.5$, and $1.0 \% \mathrm{w} /$ $\mathrm{w}$ were investigated; and for gum Arabic, 0.4, 0.5, 1.0, 5.0, $15 \% \mathrm{w} / \mathrm{w}$ were investigated. The viscosity values obtained from each of these weight percentages in the rheology tests were used in the simulation.
The grid section of the model included the use of a black oil model with dimension of 10 by 10 by 1 , a Cartesian grid, and a block-centered geometry. An initialization of the reservoir is done with a model of polymer flooding with just oil and water. The simulation was run for a period of 6 months. The reservoir was assumed to be homogenous with permeability and porosity of $50 \mathrm{md}$ and 0.2 , respectively. The dimensions of the block sizes in the grid are 75 by 75 by $30 \mathrm{~cm}$ for $\mathrm{X}, \mathrm{Y}$, and $\mathrm{Z}$ and $4000 \mathrm{~cm}$ for the top to depth face.

The PVT properties of water and dead oil are listed in Tables 1 and 2, respectively. The densities of gas, water, and oil are $0.044,64$, and $52 \mathrm{~g} / \mathrm{cc}$, respectively. A salinity of zero was used for the simulation. The solution properties and the rock properties are listed in Tables 3 and 4, respectively.

The model was initialized with solution and PVT properties, and the initial oil saturation that was obtained is shown in Figure 3. The regions with high oil saturation (up to $75 \%$ ) are 


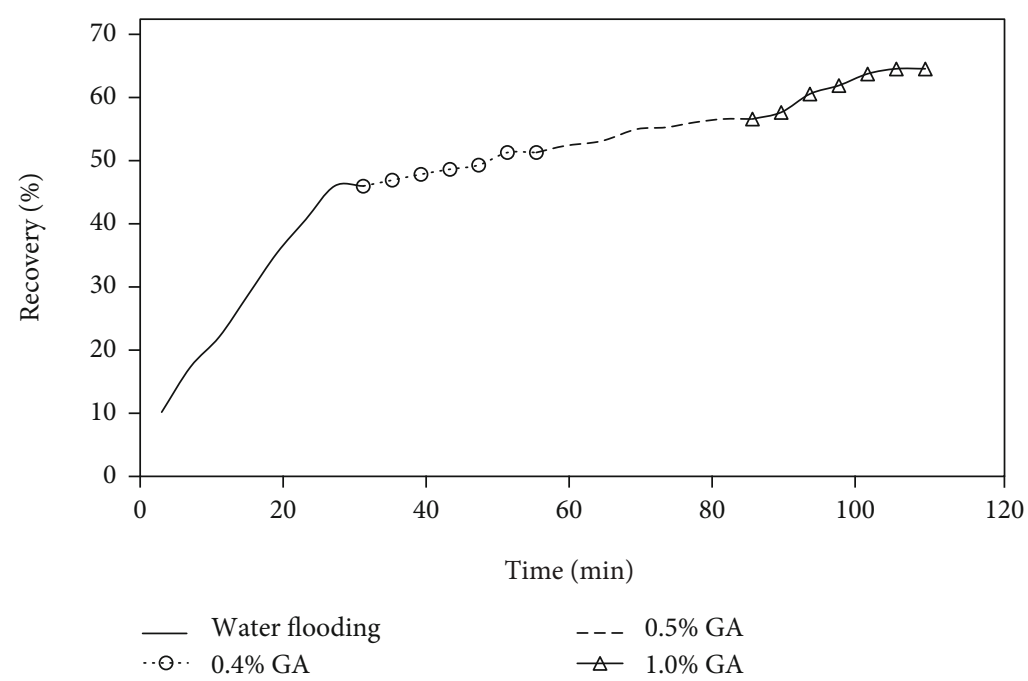

Figure 6: Total oil recovery for gum Arabic polymer.

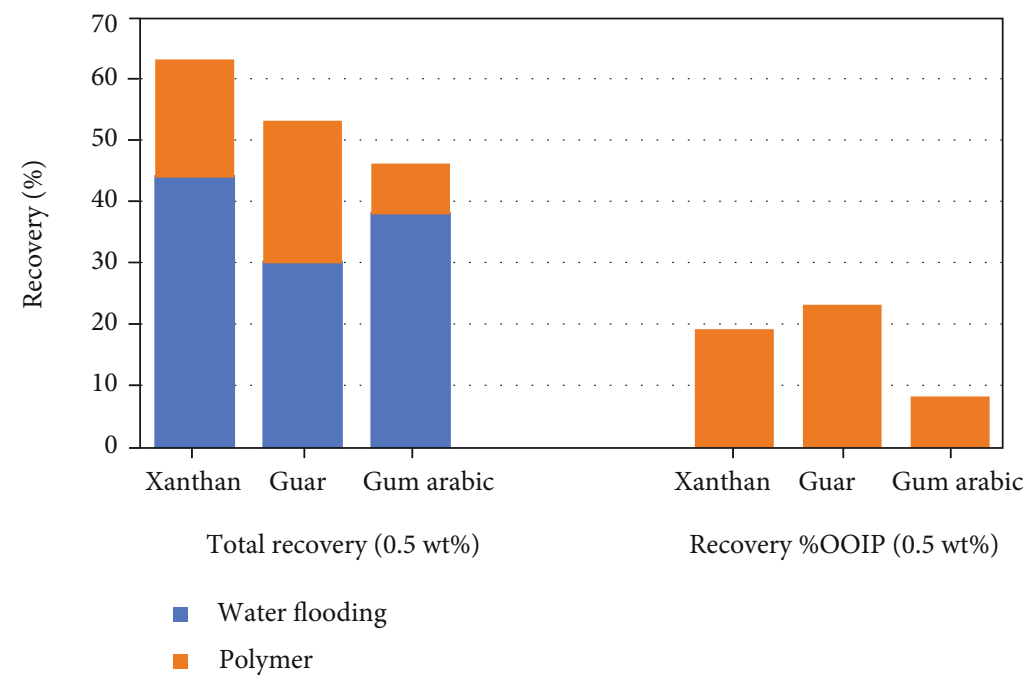

Figure 7: Recoveries of polymers at $0.5 \mathrm{wt} \%$.

shown in red. The initial oil in place was $2,641,332.3 \mathrm{cc}$, and the connate water content was $854,031.93 \mathrm{cc}$. I-injector and P-producer vertical wells were used to initialize the schedule section. The bottom pressure of the producer well was $3,999 \mathrm{~atm}$, and the water rate of the injector well was $200 \mathrm{cc} /$ $\mathrm{hr}$. To represent a typical scenario of the depletion of a reservoir, primary and secondary recoveries are first initiate and then followed by polymer flooding, but water flooding was used as the basis.

\section{Results and Discussion}

3.1. Viscosity of Polymers. The viscosity of each polymer was measured at six different weight percentages shown in Table 5. For xanthan gum and guar gum, an increase in weight percentage produced an equivalent increase in viscosity. As the weight percentage of xanthan gum and guar gum increased from 0.1 to 1.0 , the viscosity increased by 10 times. The viscosity of gum Arabic also increased with its weight percentage; however, the increase was not corresponding. As its viscosity increased by 10 times from 0.5 to 5 , its viscosity only increased by $17 \%$. It is also easily observable that gum Arabic has a low viscosity as compared to the other polymers. At $0.5 \mathrm{wt} \%$, the viscosity of xanthan gum, guar gum, and gum Arabic were 540, 410, and $170 \mathrm{cP}$, respectively. The viscosity of xanthan gum and guar gum were at least two and half times that of gum Arabic at $0.5 \mathrm{wt} \%$. At $1.0 \mathrm{wt} \%$, their viscosities were 1645,1510 , and $180 \mathrm{cP}$, respectively, with the viscosities of xanthan gum and guar gum at least 8 times that of gum Arabic. This shows that as weight percentage increases, the difference between xanthan gum and guar gum viscosities versus gum Arabic viscosity also increases.

It is believed that the increase in weight percentage increases the chances of contact between polymer molecules, 


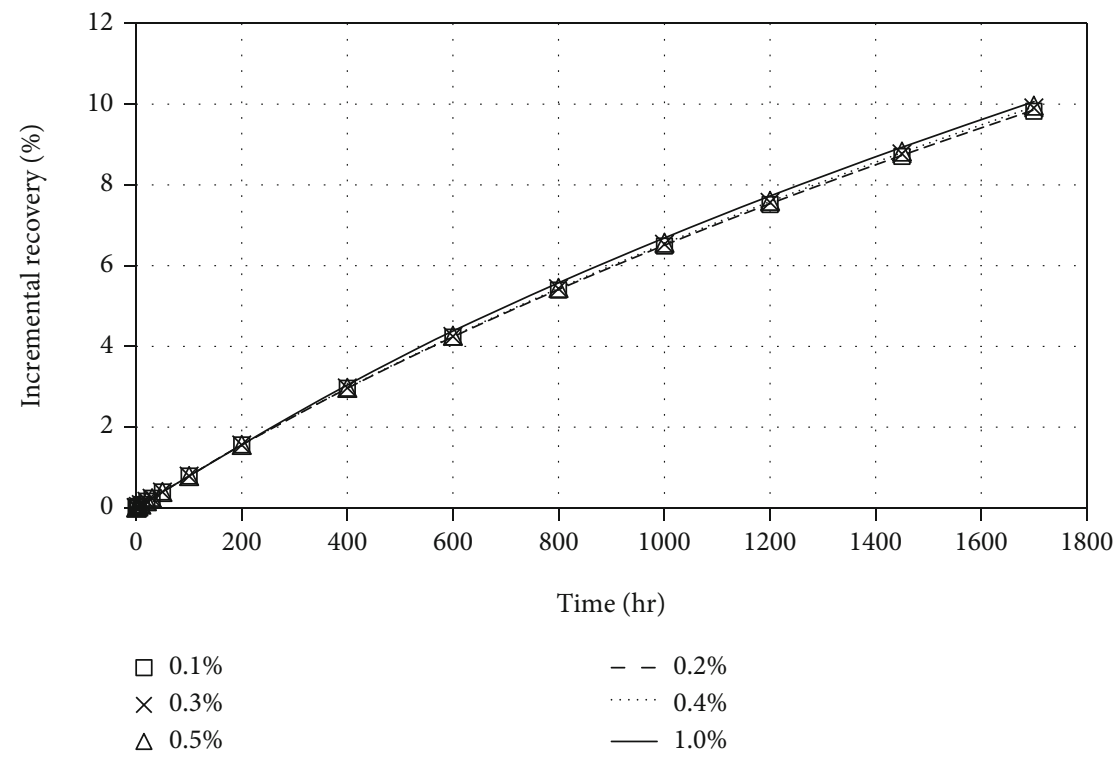

(a)

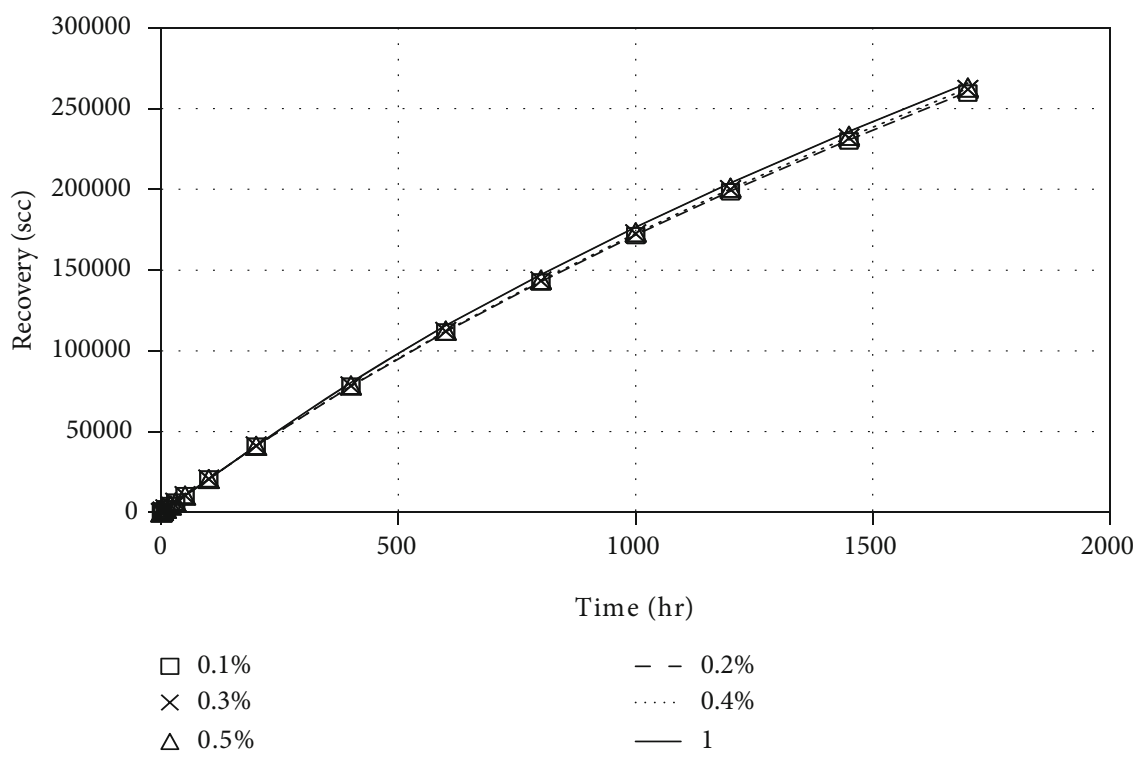

(b)

FIGURE 8: Simulated reservoir recovery for xanthan gum.

causing the viscosity of all three polymers to increase. The wide viscosity gap between gum Arabic and the other polymers is attributed to the differences in their molecular weights. The molecular weight of xanthan gum falls in the range of $2 \times 10^{6}$ to $20 \times 10^{6}$ daltons [29], and the average molecular weight of guar gum is in the range of $2 \times 10^{6}$ to $5 \times 10^{6}$ daltons [30], while the average molecular weight of gum Arabic is between $2.5 \times 10^{5}$ to $1 \times 10^{6}[31,32]$. This is consistent with the literature where it has been reported that the viscosity of xanthan gum at 1 weight percentage is higher than that of gum Arabic at 30 weight percentage; nonetheless, the viscosity of gum Arabic becomes high at high weight percentages because its structure is highly branched, giving rise to compact molecules [33]. The implications for the oil recovery potential for the polymers appear in the mobility ratios of the flooding process. Higher viscosities ensure a more uniform front for the displacing fluid which results directly in higher sweep efficiency leading to higher oil recovery. Based on this, it is expected that gum Arabic will have the lowest oil recovery, while xanthan gum and guar gum will have similar recovery capacities.

3.2. Core Flooding Experiments. Table 6 shows the petrophysical properties of the core plugs used for the flooding experiments.

The flooding results for the three concentrations of xanthan gum are shown in Figure 4. It is observed that water flooding produced $44 \%$ recovery of OOIP. 0.1 and $0.3 \%$ xanthan gum increased the oil recovery steadily, while $0.5 \%$ 


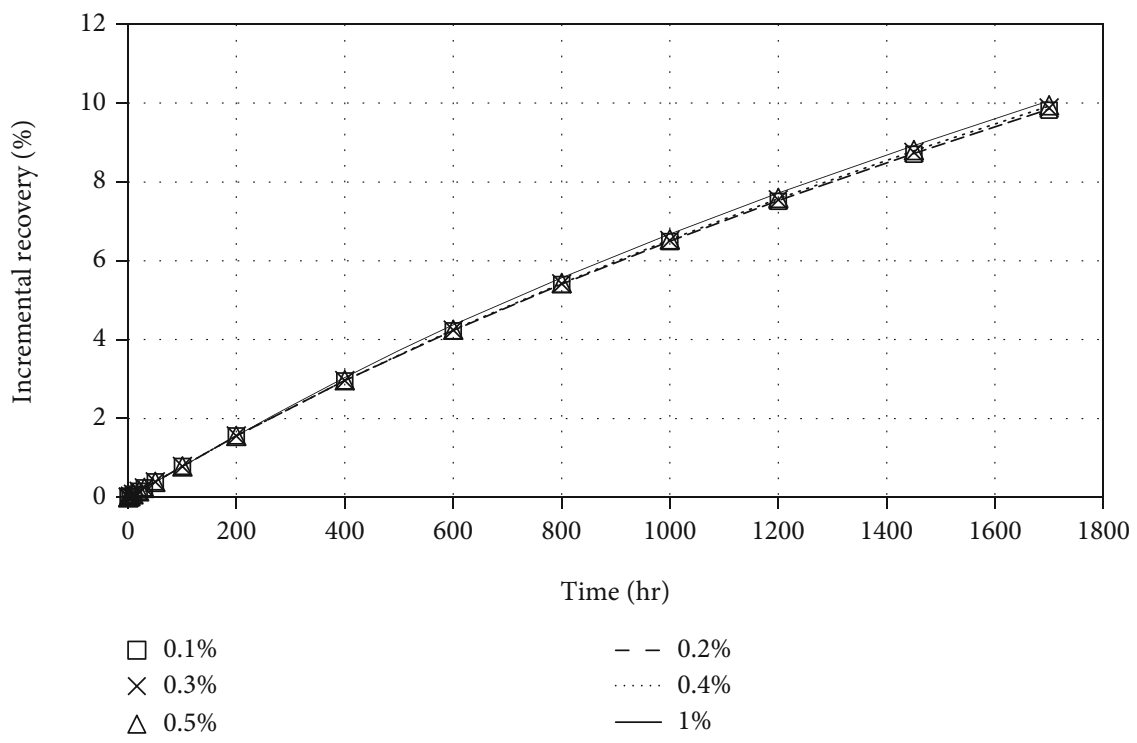

(a)

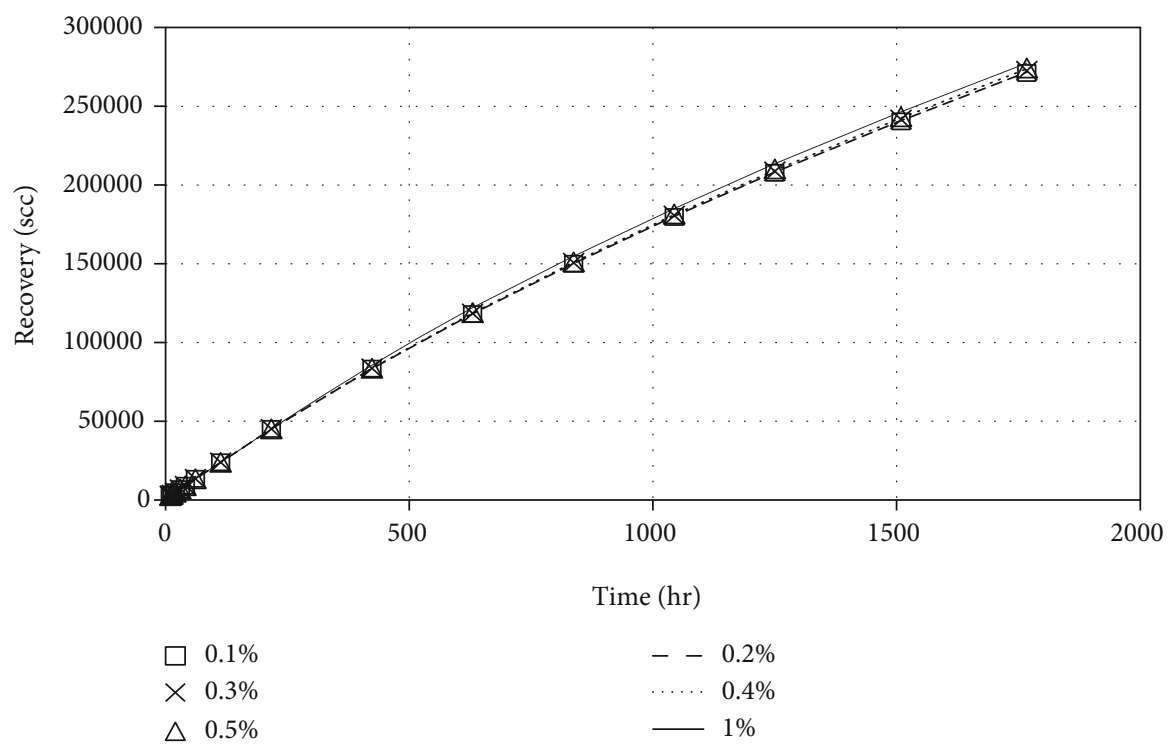

(b)

FIgURE 9: Simulated reservoir recovery for guar gum.

xanthan gum produced a sharp rise in oil recovery. In total, xanthan gum produced a total recovery of $63 \%$. Some studies in the literature have shown xanthan gum to produce incremental recovery between 9 and $18 \%$ and total recovery of up to $80 \%[19,20,34]$.

Figure 5 shows the crude oil recovery from the three weight percentages $(0.1,0.3$, and $0.5 \mathrm{wt} \%)$ of guar gum flooding done after water flooding. It was observed that water flooding recovered about $30 \%$ of OOIP. After water flooding, the sharpest increase in oil recovery was produced by $0.1 \%$ guar gum, while the 0.3 and $0.5 \%$ guar gum produced steady increases in oil recovery. In total, the total recovery achieved from the flooding process was $53 \%$, while the incremental oil recovery was $24 \%$ OOIP.

Based on total recovery, xanthan gum achieved $63 \%$ of crude oil recovery, while guar gum achieved $53 \%$. This is consistent with the results obtained from the viscosity experiments; however, a reverse is seen in the incremental oil recovery where xanthan gum achieved 19\% of OOIP while guar gum achieved 23\% of OOIP. Although the oil recovery values are close and the viscosity tests suggest that xanthan gum and guar gum have similar profiles, a higher value was expected from xanthan gum. Some of the reasons for this include the high-water flooding recovery obtained from xanthan gum flooding and the microscopic properties of the core plugs.

Water flooding and sequential gum Arabic flooding for the three weight percentages of gum Arabic (0.4, 0.5 and $1.0 \mathrm{wt} \%$ ) were conducted and shown in Figure 6. Water flooding achieved crude oil recovery of $40 \%$ OOIP. The three weight percentages of gum Arabic produced a steady increase in oil recovery up to a total of $52 \%$. Research of 


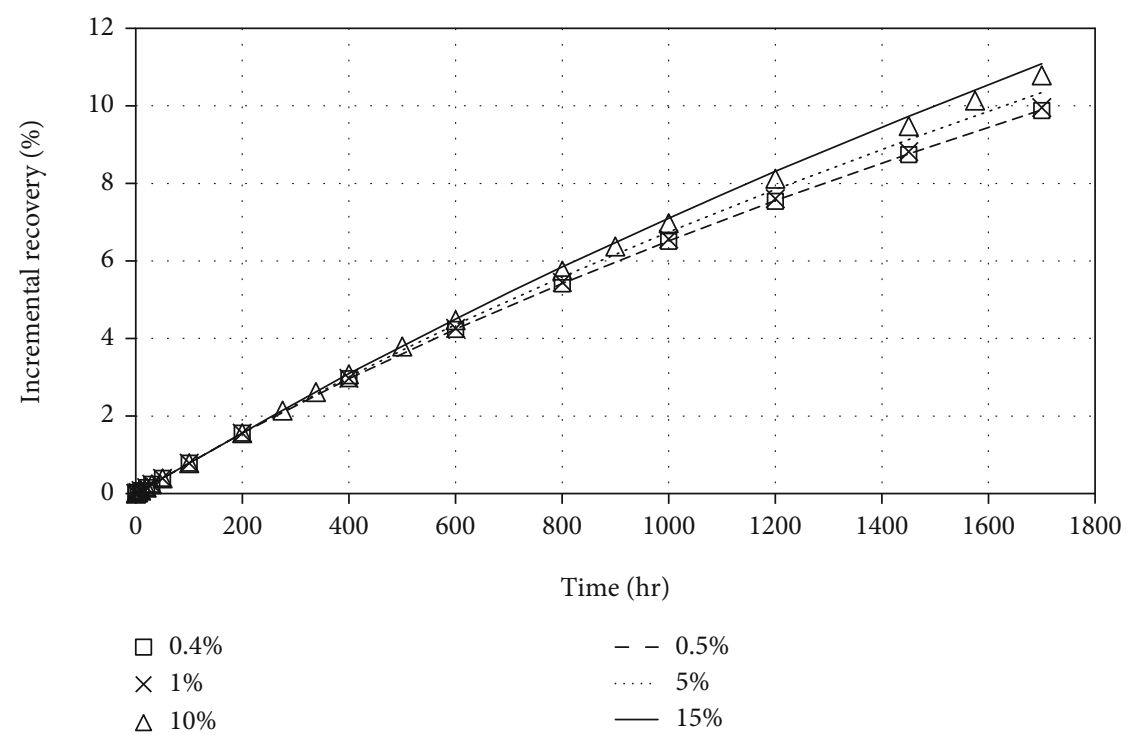

(a)

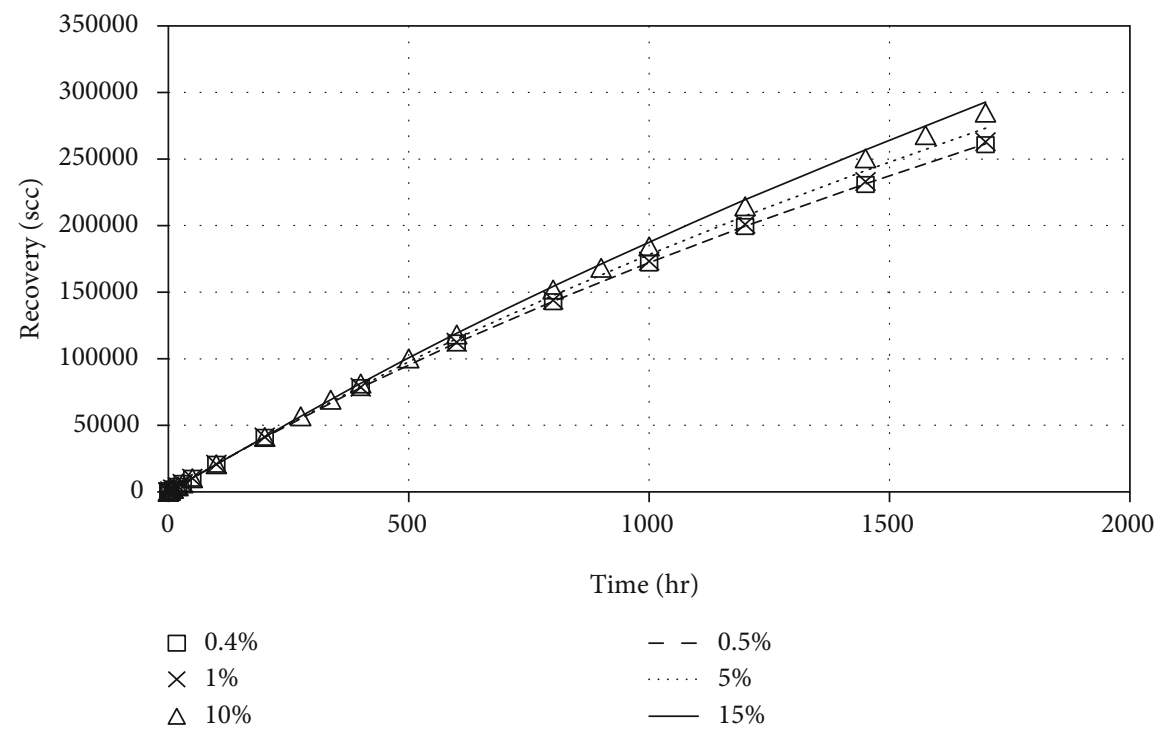

(b)

FIgURE 10: Simulated reservoir recovery for gum Arabic.

gum Arabic flooding using sandpacks achieved incremental recovery of $26 \%$ of OOIP and total recovery of $80 \%$ [34].

Xanthan gum and guar gum achieved a total recovery of 63 and 53\%, respectively, while gum Arabic achieved 52\% for the highest percentages of the three polymer used $(0.5 \%$ xanthan, $0.5 \%$ guar, and $1.0 \%$ gum Arabic). This corresponds to the results gotten from the rheology experiments. To deepen the analysis, the oil recovery at $0.5 \mathrm{wt} \%$ for all three polymers is shown in Figure 7. The observation with xanthan gum and guar gum remains the same, but the difference from the recovery of gum Arabic is further increased, thereby strengthening the results from the rheology tests.

3.3. Simulation Results. Reservoir simulation was conducted for xanthan gum, guar gum, and gum Arabic flooding using Schlumberger Eclipse ${ }^{\circledR}$ software. The OOIP and water flood- ing recoveries for the three polymers are 2,641,332 and $258,937.64 \mathrm{scc}$, respectively. Figures $8-10$ show the recovery obtained from xanthan gum, guar gum, and gum Arabic, respectively, from the simulation process. For clarity, the data are presented in Table 7.

The simulation is more reflective of the actual reservoir flooding process and will not produce the same results as the experiments because it accommodates more field parameters which could not be accounted for in the experiments. Also, the porosity and permeability of the reservoir were fixed, creating a better comparison among the polymers. For xanthan gum, guar gum, and gum Arabic, oil recovery increased with weight percentage of the polymer. This validates the result that was obtained in the core flooding experiments. Looking across the polymers, xanthan gum produced $262,424 \mathrm{scc}$ of oil at $0.4 \mathrm{wt} \%$ and guar gum produced $262,201 \mathrm{scc}$ of oil at $0.4 \mathrm{wt} \%$, 
TABLE 7: Oil recovery values for the polymers.

\begin{tabular}{lccccccc}
\hline \multirow{2}{*}{ wt\%* } & \multicolumn{2}{c}{ Xanthan gum } & \multicolumn{2}{c}{ Guar gum } & \multicolumn{2}{c}{ Gum Arabic } \\
& Oil produced (scc) & Recovery $(\%)$ & Oil produced (scc) & Recovery (\%) & wt\% & Oil produced (scc) & Recovery (\%) \\
\hline 0.1 & $259,530.84$ & 9.83 & $259,504.72$ & 9.82 & 0.4 & $260,839.91$ & 9.88 \\
0.2 & $260,340.00$ & 9.86 & $260,190.59$ & 9.85 & 0.5 & $261,441.00$ \\
0.3 & $261,726.19$ & 9.91 & $261,037.50$ & 9.88 & 1 & $263,036.91$ & 9.90 \\
0.4 & $262,424.22$ & 9.94 & $262,201.97$ & 9.93 & 5 & $273,219.88$ & 10.96 \\
0.5 & $263,075.25$ & 9.96 & $262,739.50$ & 9.95 & 10 & $284,861.94$ & 10.78 \\
1 & $265,742.69$ & 10.06 & $265,712.25$ & 10.06 & 15 & $292,827.16$ & 11.09 \\
\hline
\end{tabular}

scc: standard cubic feet.

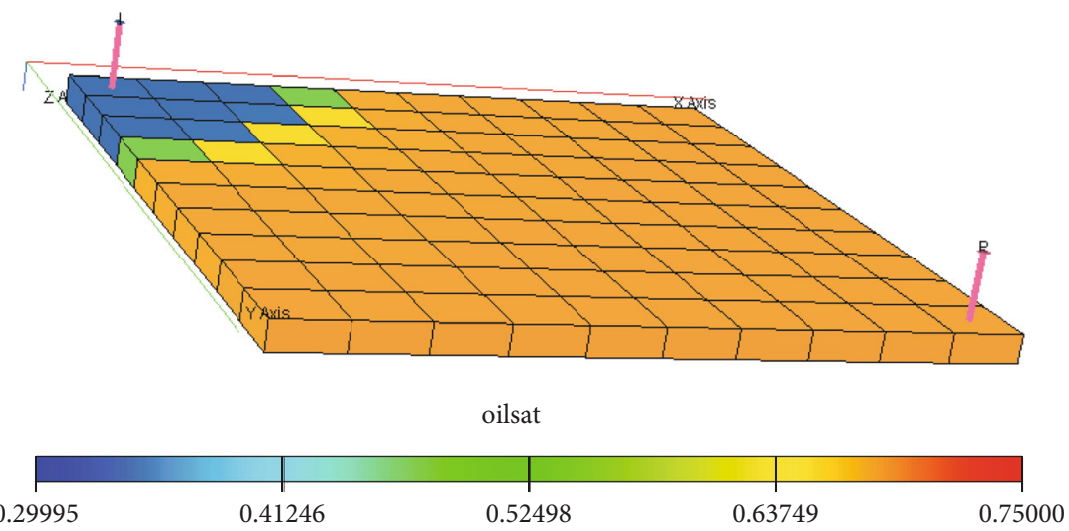

Figure 11: Final depleted model after 15 wt\% gum Arabic flooding.

while gum Arabic produced 260,839 scc of crude oil at $0.4 \mathrm{wt} \%$; with xanthan gum being the highest and gum Arabic being the lowest, the flooding experiments are further validated, and the viscosity experiments are supported by these results. Figure 11 shows the final oil saturation of the model. Comparing the figure to Figure 1, it is seen that the oil saturation has drastically reduced from 0.75 (red coloration in Figure 2) to around 0.7 in Figure 9. The effect of the polymer flooding is more seen around the injector well as the oil saturation reduced to as low as 0.5 .

\section{Conclusion and Recommendation}

The experimental results show that at the same weight percentages, xanthan gum had the highest viscosity, closely followed by guar gum. This indicates that polymers used can increase the viscosity of the displacing fluid as shown in the rheological experiment, and this correlated with the oil recovery from the core flooding experiment. At the same weight of $0.5 \%$, xanthan, guar, and Arabic gums recorded a 63\%, 53\%, and $46 \%$ oil recovery, respectively. The reservoir simulation results also validated the experimental results as at the same weight percentage of $0.5 \%$, xanthan, guar, and Arabic gum gave a recovery of $9.96 \%, 9.95 \%$ and $9.90 \%$, respectively. It is necessary to find the optimum polymer concentration that will yield the most economic oil recovery to reduce the costs of polymer injection. Biodegradable polymers such as described by Olabode et al. [15] can also be experimented to compare with other bioand non-biopolymers. The advent of nanofluids and polymer nanoparticles (PNP) has helped to increase polymer effective- ness and reduce its adsorption rates in reservoirs for enhanced oil recovery (Nezhad et al., [35], Cheraghian et al., [1] \& [36]).

\section{Nomenclature}

Wt.: Weight

EOR: Enhanced oil recovery

FVF: Formation volume factor

PVT: Pressure volume temperature

OOIP: Oil originally in place

GA: Gum Arabic

XG: Xanthan gum

GG: Guar gum

Cp: Centipoise

IFT: Interfacial tension.

\section{Data Availability}

Data will be made available on request from the first author.

\section{Conflicts of Interest}

The authors declare that they have no conflicts of interest.

\section{References}

[1] G. Cheraghian, S. Sh. Khalili Nezhad, and S. Bazgir, "Improvement of thermal stability of polyacryl amide solution used as a nano-fluid in enhanced oil recovery process by nanoclay," International Journal of Nanoscience and Nanotechnology, vol. 11, no. 3, pp. 201-208, 2015. 
[2] A. R. Kovscek, "Emerging challenges and potential futures for thermally enhanced oil recovery," Journal of Petroleum Science and Engineering, vol. 98-99, pp. 130-143, 2012.

[3] K. Moshen, M. Mohammad, and D. Amin, "Screening of enhanced oil recovery techniques for Iranian oil reservoirs using TOPSIS algorithm," Energy Reports, vol. 5, pp. 529$544,2019$.

[4] D. A. Campbell, "Enhanced oil-recovery and its environmental and economic implications in the United States," Environmental Considerations, vol. 8, no. 1, pp. 5-18, 1981.

[5] G. Hirasaki, C. Miller, and M. Puerto, "Recent advances in surfactant EOR. Paper SPE 115386," in International Petroleum Technology Conference, vol. 3, Kuala Lumpur Malaysia, 2008no. 5.

[6] G. Hirasaki and D. L. Zhang, "Surface chemistry of oil recovery from fractured, oil-wet, carbonate formations," SPE Journal, vol. 9, no. 2, pp. 151-162, 2004.

[7] J. J. Taber, In Surface Phenomena in Enhanced Oil Recovery, D. O. Shah, Ed., vol. 13, Springer, US, 1981.

[8] I. Lazar, G. Petrisor, and T. F. Yen, "Microbial enhanced oil recovery (MEOR)," Journal of Petroleum Science and Technology, vol. 25, no. 11, pp. 1353-1366, 2007.

[9] H. ShamsiJazeyi, C. A. Miller, M. S. Wong, and M. James, "Polymer-coated nanoparticles for enhanced oil recovery," Journal of Applied Polymer Science, vol. 131, no. 15, 2014.

[10] A. Rabiei, M. Sharifinik, A. Niazi, A. Hashemi, and S. Ayatollahi, "Core flooding tests to investigate the effects of IFT reduction and wettability alteration on oil recovery during MEOR process in an Iranian oil reservoir," Applied Microbial And Cell Physiology, vol. 97, no. 13, pp. 59795991, 2020.

[11] R. D. Sydansk and L. Romero-Zeron, Reservoir Conformance Improvement, Society of Petroleum Engineers, Richardson, TX, 2011.

[12] Y. Alzahid, P. Mostaghimi, M. E. Warkiani, R. T. Armstrong, V. Joekar-Niasar, and N. Karadimitriou, "Alkaline surfactant polymer flooding: what happens at the pore scale?," in $S P E$ Europec featured at 79th EAGE Conference and Exhibition, France, 2017.

[13] A. A. Olajire, "Review of ASP EOR (alkaline surfactant polymer enhanced oil recovery) technology in the petroleum industry: Prospects and challenges," Energy, vol. 77, no. 1, pp. 963-982, 2014.

[14] G. Zerkalov, Polymer Flooding for Enhanced Oil Recovery, Submitted as Coursework for PH240, Stanford University, 2015.

[15] O. A. Olabode, T. Ojo, T. Oguntade, and D. Oduwole, "Recovery potential of biopolymer (B-P) formulation from Solanum tuberosum (waste) starch for enhancing recovery from oil reservoirs," Energy Reports, vol. 6, pp. 1448-1455, 2020.

[16] A. S. Fadairo, G. Adeyemi, O. Temitope et al., "Banana peel and mango kernel-based polymers and their suitability in enhanced oil recovery," Journal of Petroleum Exploration and Production., vol. 11, no. 4, pp. 2027-2037, 2021.

[17] S. Qiao, Q. Liu, X. Zhang, and H. Che, "Syntheses of a hyperbranched polymer and its performance on enhanced oil recovery," Frontiers in Chemistry, vol. 9, 2021.

[18] G. F. Sancet, M. Goldman, J. M. Buciak et al., "Molecular structure characterization and interaction of a polymer blend of xanthan gum-polyacrylamide to improve mobility-control on a mature polymer flood," in SPE EOR Conference at Oil and Gas West Asia, Muscat, Oman, 2018.

[19] Y. Li, L. Xu, H. Gong, B. Ding, M. Dong, and Y. Li, "A microbial exopolysaccharide produced by Sphingomonas species for enhanced heavy oil recovery at high temperature and high salinity," Energy \& Fuels, vol. 31, no. 4, pp. 39603969, 2017.

[20] M. R. Couto, E. J. Gudiña, D. Ferreira et al., "Characterization of a biopolymer produced by Arthrobacter viscosus CECT 908 for application in microbial enhanced oil recovery," in Paper presented at the SPE EOR Conference at Oil and Gas West Asia, Muscat, Oman, 2018.

[21] A. Samanta, K. Ojha, and A. Mandal, "Enhanced recovery of oil by eco-friendly natural surfactant and polymer," in Proceedings of the Recent Trends in Engineering \& Education, NITTTR, pp. 28-29, Kolkata, India, 2010.

[22] L. Chen, Emulsifiers as Food Texture Modifiers Modifying Food Texture, Elsevier, USA, 2015.

[23] O. Oluwasanmi, G. Egeonu, T. Ojo, and O. Bamigboye, "Predicting the effect of well trajectory and production rates on concurrent oil and gas recovery from thin oil rims," in IOP Conference Series: Materials Science and Engineering, vol. 413, Ota, Nigeria, 2018.

[24] O. Oluwasanmi, E. Etim, O. Emeka, O. Tope, and A. Victoria, "Predicting post breakthrough performance of water and gas coning," International Journal of Mechanical Engineering and Technology, vol. 10, no. 2, 2019.

[25] T. Amirbayov, Simulation Study of the Polymer Flooding, Norwegian University of Science and Technology (Department of Petroleum Engineering), Trondheim, 2014.

[26] A. A. Manzoor, "Modeling and simulation of polymer flooding with time-varying injection pressure," American Chemical Society, vol. 5, no. 10, pp. 5258-5269, 2020.

[27] O. Olabode, D. Alaigba, D. Oramabo, and O. Bamigboye, "Modelling low-salinity water flooding as a tertiary oil recovery technique," Modelling and Simulation in Engineering, vol. 2020, Article ID 6485826, 9 pages, 2020.

[28] O. Oluwasanmi, O. Osabuhien, O. Emmanuela, and B. Felix, "The effect of chemically enhanced oil recovery on thin oil rim reservoirs," Journal of Petroleum Exploration and Production Technology, vol. 11, no. 3, pp. 1461-1474, 2021.

[29] F. Garcia-Ochoa, V. E. Santos, J. A. Casas, and E. Gomez, "Xanthan gum: production, recovery, and properties," Biotechnology Advances, vol. 18, no. 7, pp. 549-579, 2000.

[30] R. Brady, H. N. Cheng, A. Haandrikman et al., Reduced Molecular Weight Galactomannans Oxidized by Galactose Oxidase, U.S. patent office (US20020076769A1), 2002.

[31] M. Atgie, Composition and Structure of Gum Arabic in Solution and at Oil-Water, Université de Toulouse, France, 2018.

[32] I. Gashua, An Investigation of Molecular Structure, Composition and Biophysical Properties of Gum Arabic, University of Wolverhampton, UK, 2016.

[33] P. A. Williams and G. O. Phillips, "Gum arabic," in Handbook of Hydrocolloids, G. O. Phillips and P. A. Williams, Eds., pp. 252-273, Woodhead Publishing, Sawston, UK, 2nd ed. edition, 2009.

[34] I. Eiroboyi, S. S. Ikiensikimama, B. A. Oriji, and I. P. Okoye, "Experimental investigation of the macroscopic displacement efficiency of locally sourced locust bean gum and gum Arabic," in Paper presented at the SPE Nigeria Annual International Conference and Exhibition, Lagos, Nigeria, 2019. 
[35] S. K. Nezhad and G. Cheraghian, "Mechanisms behind injecting the combination of nano-clay particles and polymer solution for enhanced oil recovery," Applied Nanoscience, vol. 6, no. 6, pp. 923-931, 2015.

[36] M. F. John, O. A. Olabode, G. I. Egeonu, and T. I. Ojo, "Enhanced oil recovery of medium crude oil (31Api) using nanoparticles and polymer," International Journal of Applied Engineering Research, vol. 12, no. 19, pp. 84258435, 2017. 